\title{
Response interference by central foils is modulated by dimensions of depression and anxiety
}

\author{
Anne L. Weldon ${ }^{1}$ (D) Q Qiawen Liu $^{2} \cdot$ Wendy Heller $^{1} \cdot$ Simona Buetti $^{1}$ \\ Published online: 10 January 2020 \\ (C) The Psychonomic Society, Inc. 2020
}

\begin{abstract}
We used a maximum-likelihood-based model selection approach to investigate what aspects of affective traits influence flanker interference in a nonaffective task. A total of 153 undergraduates completed measures of anhedonic depression, anxious arousal, anxious apprehension, and a modified flanker task with two levels of perceptual load. For central foils, the most parsimonious model included load, depression, and anxious arousal. Participants scoring low on the depression and anxious arousal scales exhibited a typical perceptual load effect, with larger interference effects observed under low perceptual load compared with high perceptual load conditions. Increased depression symptoms were associated with a reduced perceptual load effect. However, the load effect reemerged in individuals who scored high on both depression and anxious arousal scales, but to a lesser extent than those scoring low on both. This pattern of results underscores the importance of studying co-occurring affective traits and their interactions in the same sample. For peripherally presented foils, the model that only included load as a factor was more parsimonious than any of the models incorporating affective traits. These findings suggest avenues for future research and highlight the role of diverse affective symptoms on various aspects of nonemotional attentional processing.
\end{abstract}

Keywords Selective attention $\cdot$ Interference $\cdot$ Perceptual load $\cdot$ Negative affect $\cdot$ Anxiety $\cdot$ Depression

The ability to selectively attend to and filter out unnecessary information is an important characteristic of attentional control. Individuals are constantly faced with having to choose between stimuli that compete for attentional resources, and such choices are repeatedly shaped by bottom-up (e.g., stimulus properties; Forster \& Lavie, 2008) as well as topdown influences (e.g., memory; Desimone \& Duncan, 1995). A considerable amount of research in selective attention is informed by perceptual load theory (Lavie, 1995; Lavie \& Tsal, 1994), in which perceptual load is manipulated by increasing the complexity of visual stimulation in a display. For instance, in a low perceptual load condition, participants report the identity of a target letter (e.g., $X$ or $N$ ), presented among a set of homogeneous nontarget letters (e.g., five $O$ s). In contrast, in high perceptual load conditions, the target is

Simona Buetti

buetti@illinois.edu

1 Department of Psychology, University of Illinois at Urbana-Champaign, 603 E. Daniel St. M/C 716, Champaign, IL 61820, USA

2 Department of Educational Psychology, University of Illinois at Urbana-Champaign, 1310 S. Sixth St., Champaign, IL 61820, USA presented among more complex nontarget letters (e.g., $V, T$, $K)$. In addition to the target letter and nontarget letters, the display also contains a task-irrelevant stimulus (e.g., an $X$ or $N$ presented at fixation or in the periphery), henceforth referred to as a foil stimulus (Lleras, Buetti, \& Mordkoff, 2014). According to perceptual load theory, under conditions of low perceptual load the target identification task is sufficiently simple that it will leave enough attentional resources to process the foil stimulus (Lavie, 2005). In contrast, the complexity of visual information to be processed in conditions of high perceptual load depletes attentional resources, and consequently the foil stimulus will not be processed.

Attentional processing of foil stimuli results in interference, known as the flanker effect (Eriksen \& Eriksen, 1974). The flanker effect is computed as the difference in reaction time between incompatible and compatible trials. Typically, a response conflict is observed in incompatible trials because the target (e.g., $X$ ) and foil stimulus (e.g., $N$ ) activate different responses (e.g., press button 1 for $X$ and button 2 for $N$ ). In compatible trials, the target and foil (e.g., both $X$ s or both $N \mathrm{~s}$ ) activate the same response, leading to response facilitation. Under conditions of low perceptual load, the foil stimulus leads to consistent interference effects, whereas conditions of high perceptual load result in reduced or null interference 
effects (Forster \& Lavie, 2008; Lavie, 2005). The magnitude of the flanker effect has been proposed as a robust measure of distractibility (Forster \& Lavie, 2007; Lavie, 2010) as well as a failure of cognitive control (Forster, Robertson, Jennings, Asherson, \& Lavie, 2014; Lachter, Forster, \& Ruthruff, 2004), particularly at low perceptual load. However, the flanker effect has also been proposed as an indicator of appropriate feature selectivity and the overall functionality of the perceptual system. For example, an individual may process the foil because it matches the attentional template for the task targets, or because it is presented in a more perceptually relevant location (e.g., at fixation; Buetti, Lleras, \& Moore, 2014; Lleras et al., 2014). Thus, the magnitude of the flanker effect might instead measure failure to inhibit either task-relevant information appearing at nontarget locations (Max \& Tsal, 2015) or response activation elicited by foils (Lleras et al., 2014). Finally, it is also possible that a flanker effect of lesser magnitude represents a failure of the perceptual system to process the foil stimulus at all.

In addition, different attentional mechanisms are involved in controlling interference from distracting, nonemotional foils presented at fixation versus foils presented in the periphery (Beck \& Lavie, 2005). Foils presented at fixation cannot be avoided, thus requiring active inhibition of the prepotent responses associated with the foil, resulting in larger flanker effects and slowed reaction times. In contrast, it has been shown that the magnitude of flanker interference is reduced as eccentricity increases (Eriksen \& Eriksen, 1974). Furthermore, when flankers appear farther away in the periphery from the search array containing the target, the likelihood that the foil will capture attention depends on the size of the attentional window (e.g., Belopolsky, Zwann, Theeuwes, \& Kramer, 2007). In fact, when the attentional window is sufficiently narrowed, stimuli falling outside of this window no longer capture attention (Belopolsky \& Theeuwes, 2010; Belopolsky et al., 2007).

The present research focused on the impact of anxiety and depression symptoms on flanker interference in a nonclinical population. The flanker task included nonemotional foils (presented at fixation or in the periphery) and varying levels of perceptual load (high and low). Symptoms of anxiety and depression, such as anhedonic depression, anxious apprehension, and anxious arousal have been shown to co-occur and to have distinct cognitive, emotional, and behavioral characteristics implemented by different neural mechanisms, (e.g., Nitschke, Heller, Imig, McDonald, \& Miller, 2001), yet few studies have investigated the effects of co-occurring psychopathology symptoms on attentional performance and inhibition together.

Below, we will first review the extant literature on how attentional control is affected by depression and anxiety symptoms alone, and by the co-occurrence of these two traits. It should be noted that many of the studies described below used a categorical and/or median split approach to separate lowscoring from high-scoring individuals along these scales. This approach was preferred presumably because most studies had small samples (e.g., Bishop, 2009: $N=8$ per group; Moriya \& Tanno, 2010: $N=22$ per group; Gotlib, Krasnoperova, Neubauer Yue, \& Joormann, 2004: $N=19$ per group; Bradley, Mogg, \& Lee, 1997: $N=17$ per group). In the present study, a large sample of participants $(N=153)$ was recruited so that we could use a continuous measures approach and look at interaction effects between depression and anxiety symptoms.

\section{Depression and attentional control}

It has been well-documented that symptoms of depression, which include increased negative affect and anhedonia, significantly affect aspects of executive function (e.g., inhibition; Gotlib \& Joormann, 2010; Joormann \& Quinn, 2014; Levin, Heller, Mohanty, Herrington, \& Miller, 2007; NolenHoeksema, 2008; Snyder, 2013). Depressive symptoms are associated with an attentional bias toward negativelyvalenced words and difficulties disengaging from negatively valenced information (Baert, De Raedt, \& Koster, 2010; Bradley et al., 1997; Gotlib et al., 2004; also see Mogg \& Bradley, 2005, for a review of how these effects vary with respect to task design). Depressive symptoms are also associated with avoidance of and poorer attentional engagement toward positively valenced stimuli (for review, see Joormann \& Vanderlind, 2014).

In contrast to findings from studies reported above that used emotional stimuli, when neutral stimuli are used in a flanker task, behavioral performance has been reported to somewhat improve in individuals with depression symptoms. For example, Dillon et al. (2015) compared performance on the flanker task in a sample of 100 patients diagnosed with depression with performance from a control group of 40 healthy individuals. In their slightly modified task, the flanker stimuli (arrows) appeared for $100 \mathrm{~ms}$ before the onset of the target. Depressed individuals responded more slowly, but more accurately on incongruent trials than nondepressed individuals did, as has been observed in other similar investigations (Chiu \& Deldin, 2007; Dubal \& Jouvent, 2004; Dubal, Pierson, \& Jouvent, 2000; Holmes \& Pizzagalli, 2010; Siegle, Steinhauser, \& Thase, 2004). The authors performed computational modeling to evaluate the different contributions of prepotent responses (reflexive activation to foil identity), inhibitory control (suppression of the prepotent response), and executive control (initiation of the correct response on incongruent trials) to task performance. Modeling results suggested that executive control was slowed down, but functioning correctly, in individuals with depression. In addition, individuals with depression had a smaller prepotent response bias. This 
smaller prepotent response was thought to offset the slowed executive control, resulting in slower yet more accurate responses on incongruent trials in the depressed group. No effects of depression on inhibitory control were observed.

\section{Anxiety and attentional control}

Symptoms of anxiety have been associated with attentional biases toward emotionally valenced and threat-related stimuli (Bar-Haim, Lamy, Pergamin, Bakermans-Kranenburg, \& van IJzendoorn, 2007; Derryberry \& Reed, 2002; Eysenck, Derakshan, Santos, \& Calvo, 2007).

In addition, individuals with elevated anxiety also appear to have more attentional resources available to process neutral, irrelevant distractors when present. Indeed, as proposed by the attentional control theory of Eysenck et al. (2007), anxiety is thought to increase the amount of attentional resources devoted to processing all bottom-up stimulation, so as to be more responsive to threatening and emotional stimuli, whenever these appear in the world. Moriya and Tanno (2010) provided evidence consistent with this theory. These authors compared flanker interference in individuals scoring high versus low on trait social anxiety (divided by a median split). Under conditions of high perceptual load, individuals high in social anxiety showed significant flanker interference, which was absent in individuals low in social anxiety. These effects were observed both when the foil was presented in the periphery (Experiment 1) and at fixation (Experiment 2). Notably, no differences between low-anxiety and high-anxiety groups were observed in the low perceptual load conditions, with both groups exhibiting similar flanker effects. This finding suggests that the impact of anxiety on flanker interference is most readily observed under conditions that require an excess of executive resources to process bottom-up information. Other studies have also reported increased interference under conditions of high perceptual load with nonemotional letter stimuli in a sample of individuals with elevated trait anxiety (Sadeh \& Bredemeier, 2011) and generalized anxiety disorder (Najmi, Amir, Frosio, \& Ayers, 2015). Taken together, these results suggest that individuals high in anxiety are generally more alert to stimuli and have more attentional resources available to process foil stimuli. From a different perspective, these results might suggest that under conditions where executive control is needed to find the target (e.g., high perceptual load conditions), anxious individuals have greater difficulty suppressing the prepotent activation associated with the foil.

In contrast to these behavioral findings, an fMRI study by Bishop (2009, $N=17$ ) found no associations between state or trait anxiety on response time or flanker interference when anxiety measures were considered as continuous variables. When participants were divided by a median split into high and low anxious individuals (using trait anxiety scores), high anxious individuals exhibited a flanker interference effect in the low load condition, whereas low anxious individuals did not (which is unusual; see Lavie, Hirst, de Fockert, \& Viding, 2004). Neither group showed flanker effects under high load conditions. Furthermore, in the low (but not high) perceptual load condition, trait anxiety was inversely associated with dorsolateral prefrontal cortex (DLPFC) activation on incongruent trials compared with congruent trials. These findings suggest that anxiety might interfere with the recruitment of control mechanisms in prefrontral regions in situations where there is an excess of resources, and where there are salient stimuli (i.e., foils) competing for attention. The underrecruitment of prefrontal regions would then be responsible for the lack of inhibitory control that is otherwise needed to suppress foil-related activation. In sum, there are mixed results in the literature studying the impact of anxiety on flanker interference, with a majority of studies finding effects of anxiety only when perceptual load is high and one reporting effects of anxiety only when perceptual load is low.

It is worth noting that many studies of anxiety and attention, including those mentioned above, do not distinguish types of anxiety, specifically anxious arousal or anxious apprehension, which have been shown to capture two separable dimensions of anxiety (e.g., Nitschke et al., 2001). Anxious arousal reflects somatic responses to stimuli (i.e., physiological arousal, hypervigilance; Clark \& Watson, 1991; Watson, Weber, et al., 1995), whereas anxious apprehension characterizes the experience of worry regarding future outcomes and includes cognitive processes that are distinguishable from rumination (Fresco, Frankel, Mennin, Turk, \& Heimberg, 2002; Hur, Heller, Kern, \& Berenbaum, 2016; Nolen-Hoeksema, 2008). Both anxious arousal and anxious apprehension are associated with unique sets of symptoms as well as with activity in distinct neural circuits (Engels et al., 2007; Engels et al., 2010; Heller, Nitschke, Etienne, \& Miller, 1997; Nitschke, Heller, Palmieri, \& Miller, 1999). These subtypes of anxiety are also associated with unique impairments in cognitive control. Whereas anxious arousal is associated with disrupted performance on spatial working memory tasks and increased hypervigilance toward potential threats, anxious apprehension is associated with disrupted performance on nonemotional working memory tasks through interfering with phonological processing (for review, see Moran, 2016).

It is therefore possible that the mixed results observed in the literature described above regarding the impact of anxiety on flanker interference may in part be due to a lack of a differentiation between anxiety subtypes. One must also consider the possibility that some of the effects reported in the literature may be false positives stemming from the small sample size and the median split procedures (e.g., Maxwell \& Delaney, 1993; Vargha, Rudas, Delaney, \& Maxwell, 1996; for various shortcomings associated with median splits, see also Brown \& 
Barlow, 2005; Cohen, 1983; Humphreys, 1978; Humphreys \& Fleishman, 1974; MacCallum, Zhang, Preacher, \& Rucker, 2002; Peters \& Van Voorhis, 1940).

\section{Combined effects of depression and anxiety}

The studies examining the effects of depression or anxiety levels on selective attention have often done so without assessing for the effects of other types of psychopathology that often co-occur with one another. It is therefore possible that a better picture might emerge when both depression and anxiety are considered, and when anxiety subtypes are taken into account. Indeed, symptoms of depression and anxiety were shown to differentially affect aspects of attention and cognitive control. For instance, Snyder et al. (2014) demonstrated that symptoms of anxiety and depression can have opposite effects when individuals must choose among several possible responses. The authors reported that when controlling for depression symptoms, elevated anxiety was associated with impaired response selection in three language tasks. This finding provides additional evidence that, even in nonaffective tasks, elevated anxiety is associated with reduced inhibition in the presence of competing response options. In contrast, when controlling for anxiety symptoms, increased depression facilitated selection. In addition, among participants with clinically elevated levels of anxiety, higher depression symptoms were still associated with facilitated selection. These patterns of results highlight the importance of considering both anxiety and depression simultaneously.

A significant amount of research has investigated the effects of both depression and anxiety symptoms on attentional control with neuroimaging. Symptoms of depression and anxiety are associated with abnormal functional activity in several brain regions that have been implicated in attention control, including the DLPFC, anterior cingulate cortex (ACC), amygdala, and inferior frontal gyrus (IFG) (Crocker et al., 2012; Engels et al., 2007; Engels et al., 2010; Levin et al., 2007; Warren et al., 2013). For example, higher levels of depressive symptoms have been associated with greater activation in the amygdala coupled with reduced activation in left DLPFC and rostral ACC in response to emotional words and faces across different experimental paradigms (Iordan, Dolcos, \& Dolcos, 2013; Levin et al., 2007; Spielberg et al., 2012). Increased activation in left IFG and reduced activation in the bilateral medial frontal gyrus has also been observed when viewing negatively-valenced words (Engels et al., 2007). Taken together, these findings suggest that symptoms of depression and anxiety are associated with patterns of brain activation indicative of dysregulated top-down attentional control in the presence of both emotional and nonemotional stimuli (for review, see Sharp, Miller, \& Heller, 2015).
In addition, unique combinations of anxious arousal, anxious apprehension, and anhedonic depression exhibited divergent activation patterns in similar brain regions during tasks of attentional and inhibitory control. For example, when comparing interference between negative and neutral words during an emotion-word Stroop task, anxious arousal and anxious apprehension moderated the effects of anhedonic depression on activation in the right DLPFC and right lateral occipital cortex, respectively, (Engels et al., 2010). Specifically, higher anhedonic depression was associated with increased activation in the right DLPFC only when anxious arousal was elevated. Higher anhedonic depression was also associated with increased activation in the right lateral occipital cortex only when anxious apprehension was elevated. This rightward bias in neural activity may indicate increased reactivity and greater allocation of attentional resources toward negatively valenced words (Engels et al., 2010). Furthermore, higher anhedonic depression combined with high anxious arousal was also associated with decreased activation in the left DLPFC and right IFG, but only when anxious apprehension was low. This suggests that a combination of high anhedonic depression and anxious arousal may bias attention by suppressing the right IFG, a region also implicated in inhibitory control (Engels et al., 2010).

\section{Present study}

The literature reviewed above highlights the role anxiety and depression can play on attentional control and the importance of also considering how the presence of these two affective traits together might uniquely affect attentional control. This is all the more important given the high level of comorbidity of depression and anxiety disorders in the general population, with 12-month comorbidity estimates ranging from $11 \%$ $35 \%$ and lifetime comorbidity estimates ranging from $10 \%$ $52 \%$ in community samples (Rusico \& Khazanov, 2017). Here, we evaluated three different affective traits and studied their impact on a modified flanker task (Beck \& Lavie, 2005), in which perceptual load was manipulated by varying the complexity of the search displays. Set size was held constant during the experiment. This practice reduces concerns proposed by dilution theory (Tsal \& Benoni, 2010), which states that changing set sizes alters the degree of dilution created by the nontarget letters. Note that the present study only manipulated perceptual load and not cognitive load (see Lavie et al., 2004). Finally, following Beck and Lavie's (2005) design, the location of the foil was manipulated between blocks of trials: During one half of the experiment, the foil was presented at fixation, where it produces the largest interference effects. During the other half of the experiment, the foil is presented in the periphery, beyond the search array, where the foil has a much smaller impact on performance. 
The impacts of anhedonic depression, anxious arousal, and anxious apprehension on varying perceptual load conditions were evaluated for centrally and peripherally presented foils. As foils appearing at fixation are always within the attention span of the task, analyses of these conditions may reveal individual differences in inhibiting responses activated by foils. In contrast, foils appearing in the periphery might create effects that depend on the breadth of attention. The ability to selectively filter out information appearing in the periphery is consistent with evidence that attentional space can be narrowed or broadened based on the location of information in the visual field (Ahmed \& de Fockert, 2012; Eriksen \& St James, 1986). More relevant to the present study are findings showing that a larger attentional breadth was associated with positive emotions and optimism (Basso, Schefft, Ris, \& Dember, 1996; Derryberry \& Tucker, 1994; Fredrickson \& Branigan, 2005; Gasper, 2004; Rowe, Hirsh, \& Anderson, 2007) and that a narrower attentional breadth was associated with depressive symptoms and negative mood (Basso et al., 1996; de Fockert \& Cooper, 2014; Derryberry \& Tucker, 1994). As a result, one might expect a larger impact of affective traits when foils are presented at fixation than when they appear in the periphery.

Finally, we should note that the present sample was representative of typical samples used in cognitive psychology to study selective attention, in that participants were undergraduate students enrolled in psychology courses at a major state university. As such, the results of this study are informative with regard to the possible observed variability in studies of selective attention. To the extent that subpopulations show nonstandard response interference patterns in their performance, it could easily be argued that differences across studies using similar methodologies may arise from differences in the affective profile of the sampled populations (for more on the possible heterogeneity of effects in cognitive psychology, see also Bolger, Zee, Rossignac-Milon, \& Hassin, 2019). For example, the present findings may provide an alternative explanation for so-called end-of-semester effects, which are anecdotal observations that participants might evidence somewhat different patterns of results at the end of the semester because of external pressures or changes in motivation (e.g., Ebersole et al., 2016; Grimm, Markman, \& Maddox, 2012; Nicholls, Loveless, Thomas, Loetscher, \& Churches, 2015; but also see Chan, Rajsic, \& Pratt, 2017).

\section{Method}

\section{Participants}

We conducted an a priori power analysis to compute an effect size of at least $r=.225$ with $80 \%$ power. The power analysis indicated that a sample size of 153 participants was needed to achieve this effect. The first 153 participants that met the inclusion criteria below were included in analyses. Participants were recruited from a large Midwestern university for course credit. Informed consent was obtained from all participants included in the study. Twenty-eight participants were compensated for their participation in lieu of course credit. All participants had normal color vision and normal or corrected-tonormal visual acuity. The final sample consisted of 153 participants (58\% female) with an average age of 19.4 years (age range: 18-31). Participants reported their race or ethnicity as Asian (44.4\%), White (39.9\%), Latino/a (8.5\%), Black $(5.9 \%)$, or other $(1.4 \%)$.

\section{Self-report measures}

The Anhedonic Depression (AD) and Anxious Arousal (AA) scales from the Mood and Anxiety Symptoms Questionnaire (MASQ; Watson, Clark, et al., 1995; Watson, Weber, et al., 1995) were used to evaluate depression and anxious arousal. The MASQ includes a 22-item AD subscale that assesses both reduced positive affect (e.g., felt optimistic; felt hopeful about the future; reverse-scored), and elevated anhedonia (e.g., felt like nothing was very enjoyable; felt withdrawn from other people). ${ }^{1}$ The 17-item AA subscale assesses physiological hyperarousal (e.g., was short of breath; felt numbness or tingling in my body). Higher scores on the AD and AA subscales indicate greater symptom severity.

The 16-item Penn State Worry Questionnaire (PSWQ; Meyer, Miller, Metzger, \& Borkovec, 1990) was administered to assess AP (Brown, Antony, \& Barlow, 1992; e.g., I am always worrying about something). Higher scores indicate greater symptom severity. The MASQ-AA and PSWQ have been established as complimentary scales that capture two separable dimensions of anxiety (Nitschke et al., 2001; Sharp et al., 2015). While the use of these measures does not confer clinical diagnoses, research indicates that they are relatively accurate at distinguishing individuals with depression and/or anxiety from healthy individuals (Bredemeier et al., 2010; Fresco, Mennin, Heimberg, \& Turk, 2003; Nitschke et al., 2001). The use of multiple measures also supports a dimensional framework for understanding symptoms that tend to co-occur across depression and anxiety. Personality traits were assessed using a 50-item scale constructed from the International Personality Item Pool (IPIP; Goldberg et al., 2006), though these data are not reported in the present study. The final sample consisted of 83 participants who completed a Google Forms version of the questionnaires, and 70 participants who completed the measures in E-Prime and did not meet the exclusion criteria below. Values for any missing

\footnotetext{
${ }^{1}$ Item 38 of the MASQ ("thought about death or suicide") was not administered, so the AD score reflects the sum of 21 items.
} 
items among included participants were interpolated using the subscale average.

\section{Stimuli and apparatus}

The experiment was run on a PC, using MATLAB, and programmed in the Psychophysics Toolbox, Version 3 (Brainard, 1997; Pelli, 1997). Stimuli were presented on a 20-inch CRT monitor with a $1,024 \times 768$ resolution. The viewing distance for stimuli was $50 \mathrm{~cm}$, and participants were stabilized by a chin rest to ensure consistency across the experiment.

Stimuli were shown in light gray and were presented on a black background. All letters were presented in font type Arial. The target and distractor letters subtended $0.54^{\circ}$ (vertically) while the foil distractor subtended $0.67^{\circ}$ (vertically) of visual angle. The foil appeared 1.3 times larger than target and distractor letters. Each trial began with a small fixation circle in the center of the screen, which subtended 0.23 degrees of visual angle, and was followed by a search display of letters positioned in the form of a circle with a radius of 2 degrees. The search display contained five nontarget letters $(S, K, V, J, R$, or $O \mathrm{~s})$, and one target letter $(X$ or $N)$. In addition, a foil ( $X$ or $N$ ) was also presented on the display either in the periphery (3.5 degrees to the left or the right of fixation) or at fixation. Compatible trials occurred when the target and foil letters matched; incompatible trials occurred when the target and foil did not match.

\section{Procedure and design}

All participants completed the self-report measures on a computer before starting the flanker task. During the flanker task, a fixation circle was displayed on the center of the screen for $2,000 \mathrm{~ms}$ at the start of each trial. Then, the circular search display (containing target and nontarget letters) and the foil were presented for $200 \mathrm{~ms}$ (see Fig. 1). Participants were asked to search for the target letter $(X$ or $N)$ on the circular display of letters while ignoring the peripheral or central foil. The target letter could appear at any position on the circle. Participants reported the identity of the target letter with the right or left arrow keys, using the right and left index fingers. In low perceptual load conditions, the nontarget letters were homogeneous (i.e., all $O \mathrm{~s}$ ). In high perceptual load conditions, the nontarget letters were heterogeneous $(S, K, V, J, R)$. The foil was always an $X$ or $N$.

Participants completed a practice block of 96 trials and then two experimental blocks of 288 trials each (576 trials total). Assignment of each target letter to the response keys was counterbalanced across participants. One block of trials contained foils presented at fixation, and another block of trials contained foils presented in the periphery. Block presentation was counterbalanced across participants. Four experimental conditions were randomized within each block: (1)
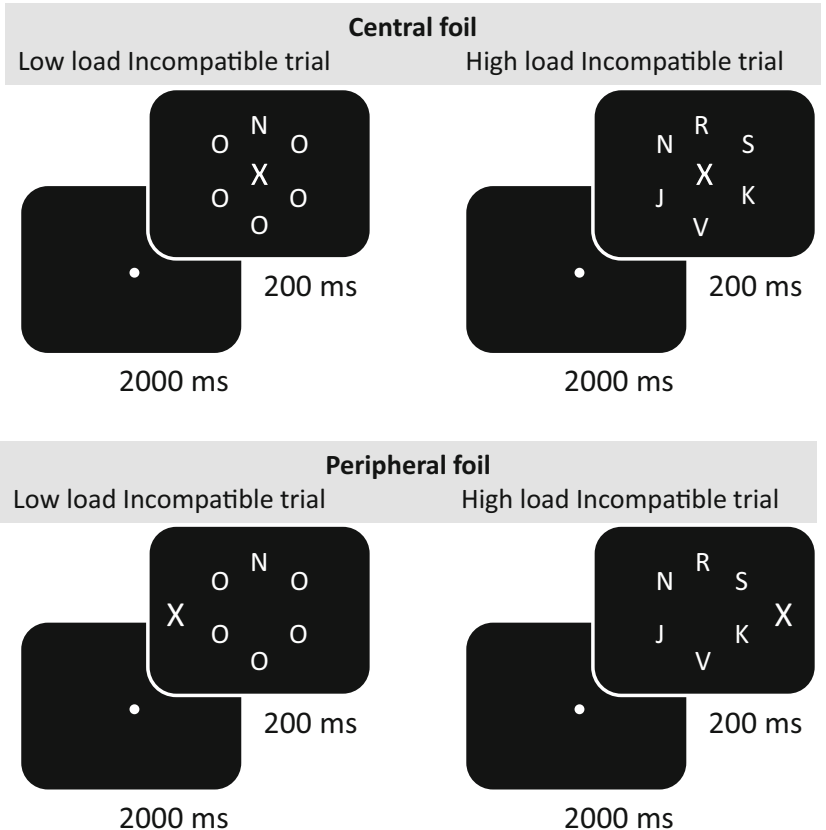

Fig. 1 Example of display (not drawn to scale) with central and peripheral foils. Examples of low and high perceptual load conditions and incompatible trials are depicted

low perceptual load with incompatible foil; (2) low perceptual load with compatible foil; (3) high perceptual load with incompatible foil; (4) high perceptual load with compatible foil.

\section{Exclusion criteria}

Participants were replaced if accuracy on the flanker task was below $70 \%(N=18)$. Participants were also replaced if they did not answer more than one item on subscales of the MASQ and PSWQ $(N=25)$, and on the trait Extraversion, Emotional Stability, or Conscientiousness subscales from the $\operatorname{IPIP}^{2}(N=26)$.

\section{Analyses}

Reaction times (RT) that were plus or minus two standard deviations from each participant's average RT for correct trials were excluded from data analysis, as were all incorrect trials ( $8.4 \%$ of trials were excluded). When testing for the distribution of the flanker effect, the Shapiro-Wilk test of normality

\footnotetext{
${ }^{2}$ Self-report measures were initially administered electronically using the EPrime 2.0 software (Psychology Software Tools, Pittsburgh, PA). A relatively large number of participants failed to respond to one or more items due to a programming flaw: Participants were instructed to press the space bar to advance to the next item, and the program did not wait for a number to be entered before advancing. When this flaw became apparent, self-report data were subsequently collected using Google Forms. Data from participants were excluded for omitting more than one item on subscales of the MASQ and PSWQ $(N=25)$ and on the trait Extraversion, Emotional Stability, or Conscientiousness subscales from the IPIP $(N=26)$. These data were not analyzed in the results reported here.
} 
indicated that the flanker effect distribution for both central and peripheral foils were not normal. This was the case for the flanker effect computed by subtraction (incompatible - compatible RTs; central foils: $\mathrm{W}=0.96036, p=2.158 \mathrm{e}-07$; peripheral foils: $\mathrm{W}=0.95638, p=6.482 \mathrm{e}-08$ ) as well as for the flanker effect computed by division (incompatible / compatible RTs; central foils: $\mathrm{W}=0.95418, p=3.43 \mathrm{e}-08$; peripheral foils: $\mathrm{W}=0.97617, p=5.697 \mathrm{e}-05$ ). The flanker effect followed an inverse Gaussian distribution (two-sided KolmogorovSmirnov for central and peripheral foils: $\mathrm{D}=0.062092, p=$ 0.5969 and $\mathrm{D}=0.071895, p=0.4077$, respectively), therefore we used the flanker effect computed by division to avoid negative numbers that are not accepted when running inverse Gaussian GLMMs. The Inverse Gaussian GLMMs with Identity link was used with the function glmer from the lme4 R package.

We used a maximum-likelihood-based model selection approach to find the most relevant variables that predict performance in the central and peripheral flanker task. All models contained the factor load as it is well established that interference changes as a function of perceptual load (e.g., Forster \& Lavie, 2008; Lavie, 2005; Lavie, 2010; Tsal \& Benoni, 2010). A total of eight models were compared to evaluate the contribution of depression alone (Model 1: Load $\times \mathrm{AD}$ ), anxiety alone (Model 2: Load $\times$ AA; Model 3: Load $\times$ AP), the interaction between anxiety factors (Model 4: $\operatorname{Load} \times \mathrm{AA} \times \mathrm{AP}$ ), and the interactions between depression and anxiety dimensions (Model 5: Load $\times \mathrm{AD} \times \mathrm{AA}$; Model 6: Load $\times \mathrm{AD} \times \mathrm{AP}$; Model 7: $\mathrm{Load} \times \mathrm{AD} \times \mathrm{AA} \times \mathrm{AP})$. A model containing the effect of load alone was also included (Model 0: Load).

For each subset of models, the most parsimonious model was identified as the model with the highest Akaike weight $\left(\mathrm{w}_{\text {model }}\right)$ computed from AIC values (Wagenmakers \& Farrell, 2004). Normalized probabilities were then computed between individual models and the best-fitting model within each subset to quantify the likelihood that the preferred model was indeed the most parsimonious given the data and model subset, $\left(\frac{w_{\text {best }}}{w_{\text {best }}+w_{i}}\right)$ (Wagenmakers \& Farrell, 2004). Chi-square tests using log-likelihood were also reported. Finally, we also estimated marginal and conditional $R^{2} . R^{2}$ quantifies the proportion of variance explained by a model, with the marginal $R^{2}$ estimating the variance accounted for by the fixed effects and the conditional $R^{2}$ estimating the variance accounted for by fixed and random effects.

\section{Results and discussion}

\section{Questionnaires}

Raw scores from questionnaire data (see Table 1, Fig. 2) were transformed into $Z$ scores.

\section{Analyses on central foils}

Model selection Maximum-likelihood based selection among the eight tested models indicated that the model including the interaction between load, depression, and anxious arousal (Model 5) was the most parsimonious among all considered models $\left(\mathrm{w} 5_{(\mathrm{AIC})}=64.1 \%\right.$; see Table 2 , top panel). The marginal $R^{2}$ for Model 5 was .101 and the conditional $R^{2}$ was .558. The second, third, and fourth most likely models were respectively: Model $1\left(\mathrm{Load} \times\right.$ depression; $\left.\mathrm{w}_{(\mathrm{AIC})}=18.2 \%\right)$, Model $0($ Load; $w 0(\mathrm{AIC})=10.6 \%)$, and Model $3(\operatorname{Load} \times$ Anxious Apprehension; w3 $3_{(\mathrm{AIC})}=2.5 \%$ ). Normalized probabilities indicated that Model 5 is $78 \%$ more likely than Model 1. Finally, chi-square tests confirmed that Model 5 is a better model than Model 1, $\chi^{2}(4)=10.52, p=.032$, and is also better than Model 0, $\chi^{2}(6)=15.60, p=.016$.

Description of the most parsimonious model The statistical effects of the most parsimonious model are presented in Table 3 (top panel).

The main effect of load was significant, confirming that the flanker effect was larger in conditions of low perceptual load (1.12) compared with high perceptual load (1.08). The difference in interference between low and high load conditions will be henceforth referred to as the perceptual load effect. Table 4 shows the mean RT and flanker effect (by subtraction and by division) per condition.

The interaction between load and depression was also significant. As illustrated in Fig. 3 (left panel), the interaction between load and depression is best characterized by a much larger impact of load on the magnitude of the flanker effect among individuals scoring low in depression compared with individuals scoring high in depression, for whom the effect of load is substantially smaller. Only the difference between the slopes was significant, as indicated by the significant two-way interaction (see Table 3), but neither the low load slope nor the high load slope differed from zero, $t(149)=-1.24, p=.216$, and $t(149)=0.427, p=.670$, respectively. This two-way interaction was further qualified by anxious arousal as described below (see Fig. 3, right panel).

The three-way interaction between load, depression, and anxious arousal was also significant (see Fig. 3, right panel). Simple slope analyses were conducted to characterize this interaction (Dawson, 2014; Dawson \& Richter, 2006). Below, we discuss the two slope comparisons that reached significance, as well as three marginal effects that relate to prior findings in the literature (Tables 5 and 6 show all the pairwise slope comparisons). To simplify the description of the results, Fig. 4 visualizes the significant and marginal effects as a function of depression (Fig. 4a and c) and anxious arousal (Fig. 4b, d, and e).

First, the results showed that when considering individuals with low anxious arousal (see Fig. 4a), as depression scores 
Table 1 Descriptive statistics of self-report measures

\begin{tabular}{llll}
\hline Psychopathology measures & Mean $(S E)$ & Observed range & Possible range \\
\hline Anhedonic depression (AD) & $44.4(1.44)$ & $23-99$ & $22-110$ \\
Anxious arousal (AA) & $39.8(0.485)$ & $17-59$ & $17-85$ \\
Anxious apprehension (AP) & $52.4(1.13)$ & $21-80$ & $16-80$ \\
\hline
\end{tabular}

increased, the effect of perceptual load decreased, $t(145)=$ $-3.74, p<.001$. This indicated that in our sample, the traditional perceptual load effect (i.e., the increase in interference in low compared with high load conditions; Lavie et al., 2004) was best observed in individuals who scored low on both depression and anxious arousal. These individuals likely have the ability to appropriately distribute attentional resources and successfully inhibit prepotent responses such that foil interference is minimized (e.g., Max \& Tsal, 2015). However, as the depression score increased, the effect of load decreased. Second, when considering individuals with higher depression scores (see Fig. 4b), the perceptual load effect was largest for those individuals who also had higher anxious arousal, $t(145)$ $=2.088, p=.039$. In sum, these two results suggest that the magnitude of the perceptual load effect was determined by an interaction between depression and anxious arousal such that a large perceptual load effect was observed for individuals with low scores on both scales, whereas a minimal perceptual load effect was observed for individuals with high scores on the depression scale and low scores on the anxious arousal scale. Furthermore, the perceptual load effect was evident for individuals with high scores on both scales. We will return to these findings in the General Discussion.

The marginal effects discussed below are relevant with respect to previous findings in the literature that investigated the effects of depression (e.g., Dillon et al., 2015) and anxiety (e.g., Moriya \& Tanno, 2010; Bishop, 2009) on selective attention, that found modulations of flanker interference at specific load levels.
First, when considering the low perceptual load condition (see Fig. 4c), the decrease in flanker interference as the level of depression symptoms increased was modulated by the level of anxious arousal: A greater reduction was observed in individuals with low scores of anxious arousal, although this effect was only marginal, $t(145)=-1.76, p=.08$. This result is reminiscent of prior findings showing that depression tends to reduce flanker interference (Chiu \& Deldin, 2007; Dillon et al., 2015; Dubal \& Jouvent, 2004; Dubal et al., 2000; Pizzagalli \& Holmes, 2009; Siegle et al., 2004). The current results suggest that in our sample, a similar pattern was observed in individuals who scored high in depression and low in anxious arousal. The result is also consistent with findings from Snyder et al. (2014) showing that after controlling for anxiety scores, increased depression scores were associated with better verbal selection among competing responses.

Second, when looking only at individuals scoring low in depression (see Fig. 4d), the perceptual load effect tended to become smaller as anxious arousal increased, $t(145)=-1.685$, $p=.094$. Specifically, anxious arousal increased flanker interference in the high-load condition, while the increase was very minimal in the low load condition, suggesting that the suppression of foils at fixation becomes more difficult for individuals with high anxious arousal and low depression (dashed line in Fig. 4d). This marginal finding is consistent with prior results in the literature examining the effects of anxiety on flanker interference (Moriya \& Tanno, 2010; Najmi et al., 2015; Sadeh \& Bredemeier, 2011). For instance, Moriya and Tanno (2010) showed that under conditions of low load,
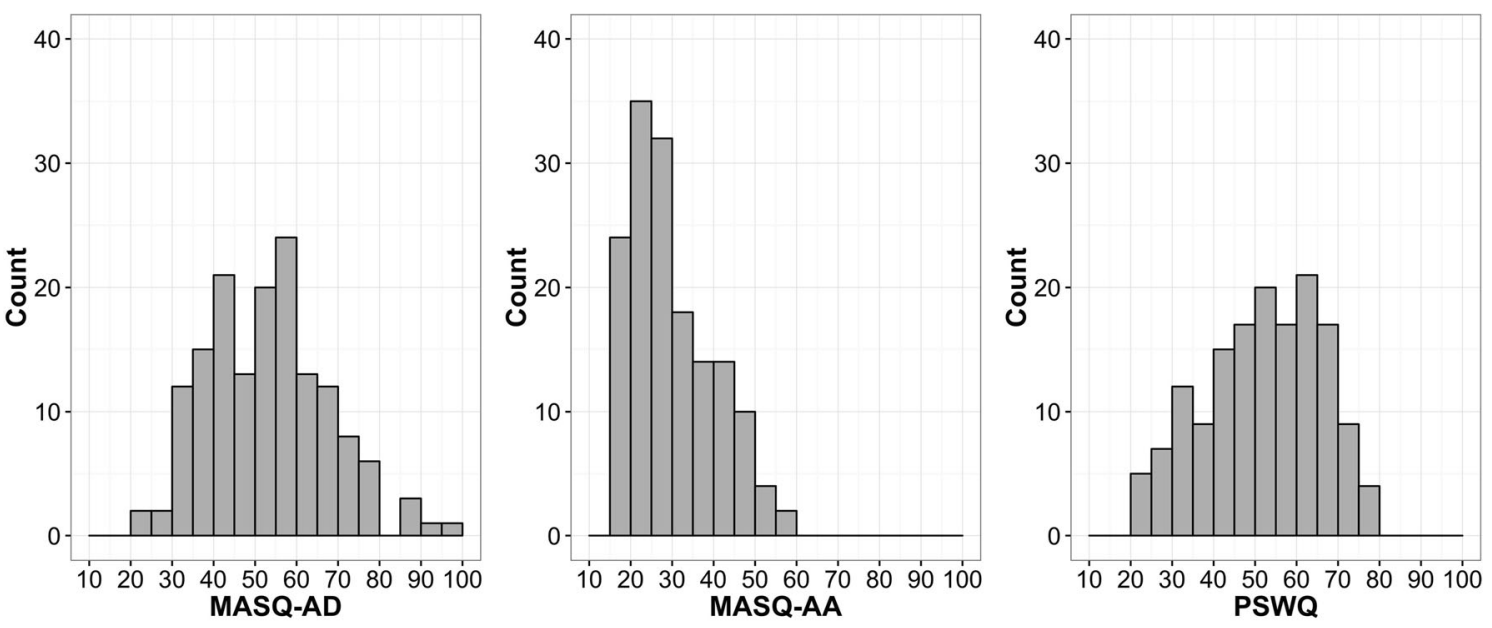

Fig. 2 Distributions of self-report measures 
Table 2 Results of the maximum-likelihood based model selection for central (top) and peripheral (bottom) foils conditions

\begin{tabular}{|c|c|c|c|c|c|c|}
\hline Model & Fixed and Random effects & Df & AIC & $\Delta_{\mathrm{i}}(\mathrm{AIC})$ & $\mathrm{w}_{\mathrm{i}}(\mathrm{AIC})$ & $\mathrm{N} \operatorname{Pr}$ \\
\hline \multicolumn{7}{|c|}{ A. Central flanker } \\
\hline 5 & Load $\times \mathbf{A D} * \mathbf{A A}+(1 \mid$ Subject $)$ & 10 & -791.38 & 0 & 0.641 & \\
\hline 1 & Load $\times$ AD $+(1 \mid$ Subject $)$ & 6 & -788.86 & 2.52 & 0.182 & 0.779 \\
\hline 0 & Load & 4 & -787.78 & 3.6 & 0.106 & 0.858 \\
\hline 3 & Load $\times$ AP + (1 | Subject $)$ & 6 & -784.89 & 6.49 & 0.025 & 0.962 \\
\hline 2 & Load $\times$ AA + (1 $\mid$ Subject $)$ & 6 & -784.44 & 6.94 & 0.020 & 0.970 \\
\hline 4 & Load $\times$ AP $\times$ AA + (1 | Subject $)$ & 10 & -784.07 & 7.31 & 0.017 & 0.975 \\
\hline 6 & Load $\times \mathrm{AD} \times \mathrm{AP}+(1 \mid$ Subject $)$ & 10 & -782.75 & 8.63 & 0.009 & 0.987 \\
\hline 7 & Load $\times \mathrm{AD} \times \mathrm{AA} \times \mathrm{AP}+(1 \mid$ Subject $)$ & 18 & -778.92 & 12.46 & 0.001 & 0.998 \\
\hline \multicolumn{7}{|c|}{ B. Peripheral flanker } \\
\hline 0 & Load & 4 & -888.38 & 0 & 0.581 & \\
\hline 3 & Load $\times$ AP $+(1 \mid$ Subject $)$ & 6 & -886.47 & 1.91 & 0.223 & 0.722 \\
\hline 2 & Load $\times$ AA + (1 | Subject $)$ & 6 & -884.82 & 3.56 & 0.098 & 0.856 \\
\hline 1 & Load $\times$ AD + (1 $\mid$ Subject $)$ & 6 & -884.45 & 3.93 & 0.081 & 0.877 \\
\hline 4 & Load $\times$ AP $\times$ AA + (1 | Subject $)$ & 10 & -880.08 & 8.3 & 0.009 & 0.984 \\
\hline 6 & Load $\times \mathrm{AD} \times \mathrm{AP}+(1 \mid$ Subject $)$ & 10 & -879 & 9.38 & 0.005 & 0.991 \\
\hline 5 & Load $\times$ AD $\times$ AA + (1 | Subject $)$ & 10 & -877.25 & 11.13 & 0.002 & 0.996 \\
\hline 7 & Load $\times \mathrm{AD} \times \mathrm{AA} \times \mathrm{AP}+(1 \mid$ Subject $)$ & 18 & -867.64 & 20.74 & 0.000 & 1.000 \\
\hline
\end{tabular}

Note. The best fitting model is shown in boldface. $\mathrm{N}$ Pr values indicate the normalized probability that the preferred model provides the best fit. AD $=$ anhedonic depression; $\mathrm{AA}=$ anxious arousal; $\mathrm{AP}=$ anxious apprehension; $d f=$ degrees of freedom; $\mathrm{AIC}=\mathrm{Akaike}$ information criteria; $\Delta_{\mathrm{i}}(\mathrm{AIC})=$ difference in AIC compared with best-fitting model; $\mathrm{w}_{\mathrm{i}}(\mathrm{AIC})=$ Akaike weights; $\mathrm{N} \operatorname{Pr}=$ normalized probability

individuals with low and high anxiety exhibited similar foil interference effects (also seen in the solid line in Fig. 4d), whereas under conditions of high load, individuals with high anxiety showed a larger flanker interference that was absent in low anxiety individuals (similar to the dashed line in Fig. 4d). Overall, this result supports Moriya and Tanno's (2010) conclusion that anxiety differentially modulates flanker interference in conditions that require an excess of executive resources to process bottom-up information (i.e., high load), but not when there is an excess of cognitive resources available to process the foils (i.e., low load). Note that this result also runs counter to the normative finding and account of perceptual load effects on foil interference (e.g., Beck \& Lavie, 2005). Indeed, in the high load condition, load theory argues that there are little attentional resources available to process the foil, and therefore interference effects are expected to be minimal. As proposed by Eysenck et al. (2007), it is possible that anxiety increases the allocation of attentional resources for processing bottom-up information, thereby leading to a larger interference effect on high load trials. Moreover,

Table 3 Summary of the most parsimonious models for central (top) and peripheral foils (bottom), indicating the main and interactions effects

\begin{tabular}{|c|c|c|c|c|}
\hline & Estimate & $S E$ & $t$ value & $p$ \\
\hline \multicolumn{5}{|l|}{ A. Central flanker } \\
\hline (intercept) & 1.086 & 0.008 & 135.186 & $<.0001$ \\
\hline Load & 0.038 & 0.006 & 6.501 & $<.0001$ \\
\hline Depression & 0.003 & 0.008 & 0.427 & .669 \\
\hline Anxious arousal & 0.008 & 0.008 & 0.997 & .319 \\
\hline Load $\times$ Depression & -0.013 & 0.006 & -2.300 & .021 \\
\hline Load $\times$ Anxious Arousal & -0.001 & 0.006 & -0.097 & .923 \\
\hline Depression $\times$ Anxious Arousal & -0.012 & 0.007 & -1.756 & .079 \\
\hline Load $\times$ Depression $\times$ Anxious Arousal & 0.015 & 0.005 & 2.954 & .003 \\
\hline \multicolumn{5}{|l|}{ B. Peripheral flanker } \\
\hline (intercept) & 1.031 & 0.006 & 164.453 & $<.0001$ \\
\hline Load & 0.0328 & 0.005 & 6.043 & $<.0001$ \\
\hline
\end{tabular}

Items that are significant at $\mathrm{p}<0.05$ are presented in boldface 
Table 4 Reaction time (ms) and standard error (in parenthesis) for incongruent and congruent conditions as a function of load (low and high) for central (top) and peripheral (bottom) foils conditions

\begin{tabular}{lllll}
\hline & Incongruent & Congruent & flanker effect subtraction & flanker effect division \\
\hline $\begin{array}{l}\text { A. Central flanker } \\
\text { Low load }\end{array}$ & $689(119)$ & $615(112)$ & $73.6(51.7)$ & $1.12(.01)$ \\
High load & $855(149)$ & $793(147)$ & $61.5(59.8)$ & $1.08(.01)$ \\
B. Peripheral flanker & & & & \\
Low load & $628(123)$ & $591(106)$ & $36.9(44.6)$ & $1.06(.01)$ \\
High load & $790(146)$ & $769(145)$ & $21.4(50)$ & $1.03(.01)$ \\
\hline
\end{tabular}

Note. The flanker effect is shown when computed by subtraction (incompatible minus compatible) and by division (incompatible divided by compatible). The standard error is shown in parenthesis

this result might also provide an additional mechanism to explain the tendency for end-of-semester participants (as a group) to show a slightly larger Stroop effect than at other points in the semester (Ebersole et al., 2016), as at this time of the semester students may be experiencing additional stressors and possibly elevated levels of anxious arousal. The elevated anxiety might lead to difficulties suppressing the prepotent response in the Stroop task.

Finally, when looking only at the low load condition (see Fig. 4e), flanker interference increased with increasing anxious arousal, but more so when individuals also scored high in depression, $t(145)=-1.756, p=.081$. This finding is reminiscent of what Bishop (2009) observed in low-load conditions: Foil interference was found only in individuals with high anxiety and not in individuals with low anxiety. Note that finding a null interference effect in low load conditions is unusual (Lavie et al., 2004). In our sample, the smallest interference in the low load condition was observed in individuals scoring low in anxious arousal and high in depression. In comparison, individuals scoring high in anxious arousal showed a larger interference effect.

\section{Analyses on peripheral foils}

Model selection and discussion of most parsimonious model Maximum-likelihood-based selection among the seven tested
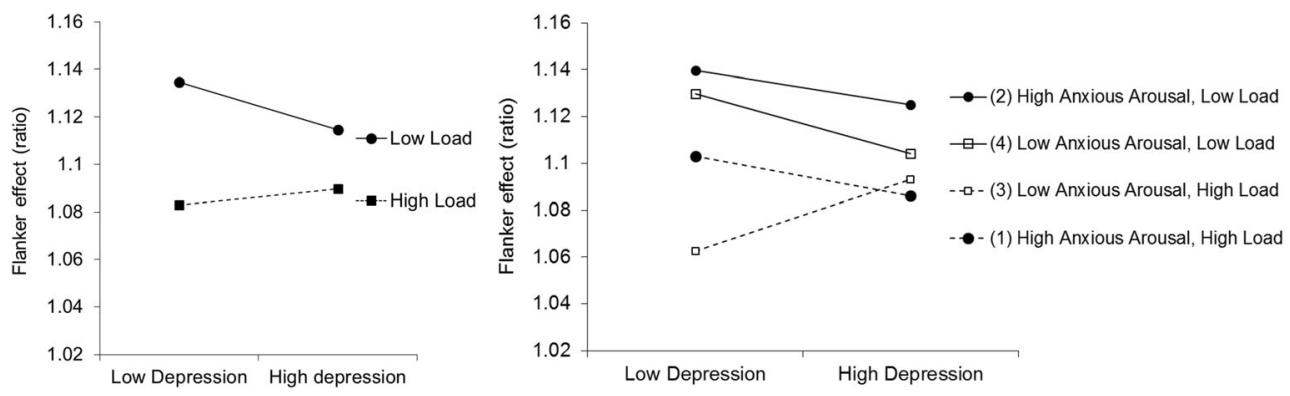

Fig. 3 Left panel: Illustration of the load by depression interaction in the most parsimonious model. The interaction is illustrated at the \pm 1 standard deviation away from the mean in the depression score. The flanker effect (ratio) was obtained by dividing RTs for incompatible trials by RTs for models indicated that the model including load only (Model 0) was the most parsimonious among all considered models (w0 $0_{\text {AIC) }}=58.1 \%$ ). The marginal $R^{2}$ for Model 0 was .059 and the conditional $R^{2}$ was $.039 \%$. The second, third, and fourth most likely models were respectively: Model 3 (Load $\times$ Anxious Apprehension; $\left.\mathrm{w}_{(\mathrm{AIC})}=22.3 \%\right)$, Model $2(\operatorname{Load} \times$ Anxious Arousal; $\left.\mathrm{w} 2_{(\mathrm{AIC})}=9.8 \%\right)$, and Model $1(\mathrm{Load} \times$ Depression; $\mathrm{w} 1_{(\mathrm{AIC})}=8.1 \%$ ). Normalized probabilities indicate that Model 0 is $72 \%$ more likely than Model 3. Finally, chi-square tests indicated that Model 0 and Model 3 did not differ from each other, $\chi^{2}(2)=2.09, p=.351$, nor from Model $2, \chi^{2}(2)=0.45, p=0.8$. The summary of Model 0 indicated that the main effect of load was significant, and flanker interference was larger in the low load than in the high load condition (1.06 vs. 1.03 ; see Table 4$)$.

\section{General discussion}

The present study used a dimensional approach to investigate whether combinations of depression and anxiety types differentially modulate flanker interference at varying levels of perceptual load. Flanker interference was evaluated both with foils presented at fixation and with foils presented in the periphery. Previous findings indicated that attentional control can be modulated by depression (Chiu \& Deldin, 2007;

compatible trials. Right panel: Illustration of the load by depression by anxious arousal interaction. Low and high anxious arousal are also estimated at the \pm 1 standard deviation away from the mean in the anxious arousal score 
Table 5 Slope difference tests for the slopes shown in Fig. 3 (right panel)

\begin{tabular}{lll}
\hline Pair of slopes & $\boldsymbol{t}$ value for slope difference & $\boldsymbol{p}$ value for slope difference \\
(1) and (2) & 0.154 & 0.878 \\
(1) and (3) & 0.408 & 0.684 \\
(1) and (4) & -1.604 & 0.111 \\
(2) and (3) & 0.316 & 0.752 \\
(2) and (4) & -1.756 & 0.081 \\
(3) and (4) & $-\mathbf{3 . 7 4 4}$ & $<\mathbf{0 . 0 0 1}$ \\
Individual slopes & $\boldsymbol{t}$ value for simple slope & $\boldsymbol{p}$ value for simple slope \\
(1) & -0.796 & 0.427 \\
(2) & -0.669 & 0.505 \\
(3) & 1.479 & 0.141 \\
(4) & -1.23 & 0.221 \\
\hline
\end{tabular}

Items that are significant at $\mathrm{p}<0.05$ are presented in boldface

Dillon et al., 2015; Dubal \& Jouvent, 2004; Dubal et al., 2000), anxiety (Moriya \& Tanno, 2010; Najmi et al., 2015; Sadeh \& Bredemeier, 2011), and their interaction (Snyder et al., 2014). A maximum-likelihood model selection approach was used to compare how flanker interference was best predicted in the presence of these affective traits. The tested models evaluated the contribution of depression alone, anxiety alone, the interaction between anxiety dimensions, and the interactions between depression and anxiety dimensions. In addition, a null model containing only the effect of load was included to understand whether adding individual difference measures improves our understanding of the variability observed in flanker interference tasks above and beyond the effect of load.

Results indicated that for foils presented at fixation, the most parsimonious model included the interaction between load, depression, and anxious arousal. The two significant interactions found for central foils demonstrate how the perceptual load effect is modulated by combinations of depression and anxious arousal scores. According to load theory (Lavie et al., 2004), the physical characteristics of a display determine the extent to which foil interference will be observed. When the display can be easily parsed (i.e., low perceptual load), there should be enough attentional resources to process both the target and the foil stimuli, leading to foil interference. In contrast, when the display cannot be easily parsed (i.e., high perceptual load), all attentional resources are devoted to finding the target, leaving little to no resources available to process the foil, which should eliminate any interference associated with the foil. The results presented here show that the prototypical perceptual load effect was in fact modulated by individual differences in depression and anxious arousal, suggesting that perceptual information alone does not determine the extent of foil interference.

The typical perceptual load effect was evident when individuals scored low on both scales of depression and anxious arousal, diminished when individuals had an elevated score on the depression scale (and a low score on anxious arousal), and reemerged when individuals had elevated scores on both scales, though to a lesser extent than those scoring low on both (see Fig. 4a-b). The fact that the perceptual load effect diminishes with increasing levels of depression might be explained by previous findings in the literature suggesting that these individuals show less of a bias to automatically orient to

Table 6 Slope difference tests for the slopes shown in Fig. 4b, d, e

\begin{tabular}{lll}
\hline Pair of slopes & $\boldsymbol{t}$ value for slope difference & $\boldsymbol{p}$ value for slope difference \\
(1) and (2) & $\mathbf{2 . 0 8 8}$ & $\mathbf{0 3 9}$ \\
(1) and (3) & 0.408 & .684 \\
(1) and (4) & -0.683 & .496 \\
(2) and (3) & -0.594 & .554 \\
(2) and (4) & -1.756 & .081 \\
(3) and (4) & -1.685 & .094 \\
Individual slopes & $\boldsymbol{t}$ value for simple slope & $\boldsymbol{p}$ value for simple slope \\
(1) & -0.385 & .701 \\
(2) & 1.132 & .259 \\
(3) & 1.661 & .099 \\
(4) & 0.397 & .692 \\
\hline
\end{tabular}



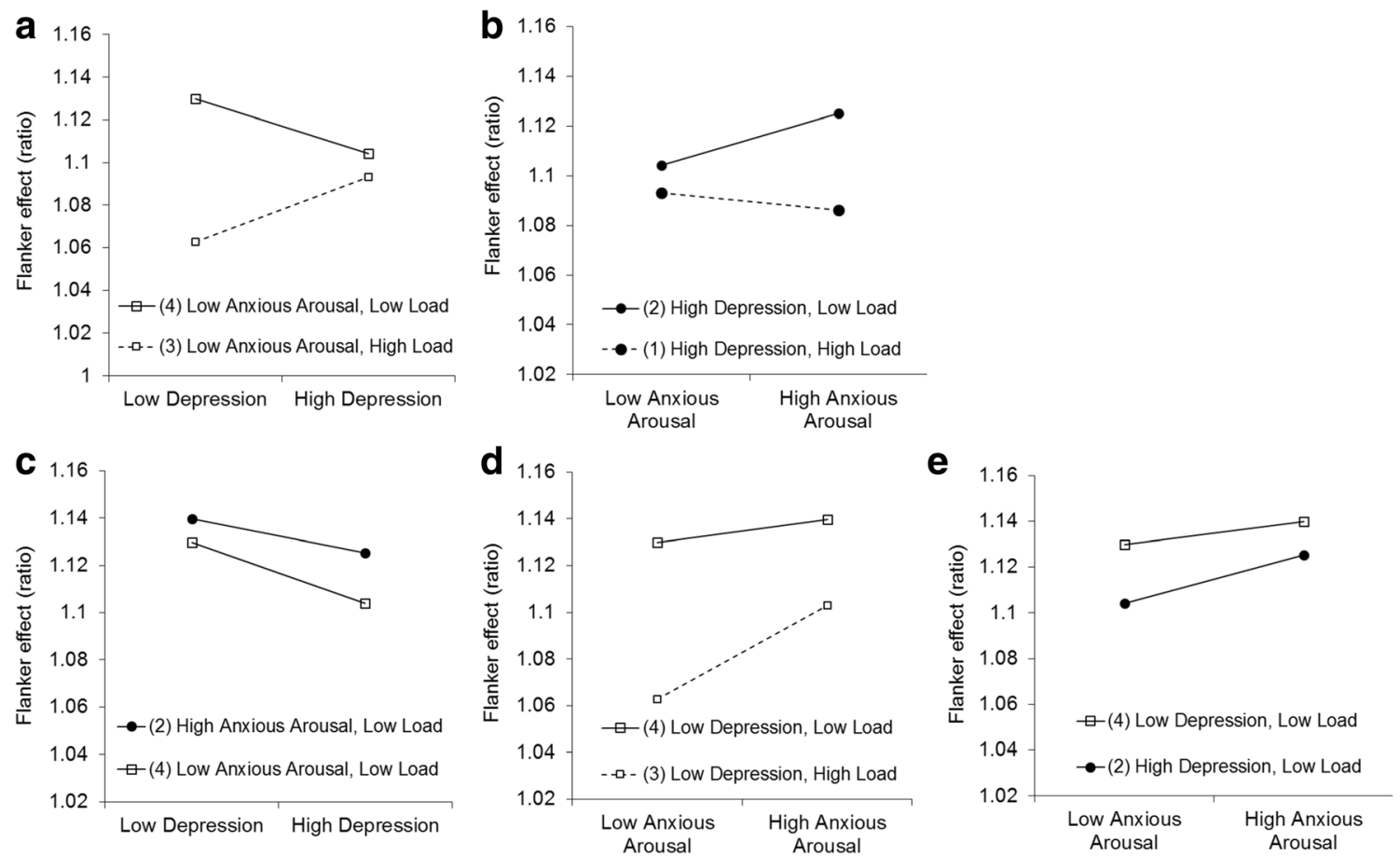

Fig. 4 Statistically significant $(\mathbf{a}-\mathbf{b})$ and marginal (c-e) slope comparisons illustrating the interaction between load, depression, and anxious arousal. Low and high points on the Depression and Anxious Arousal scales were estimated at the \pm 1 standard deviation score away from the sample mean

foils (Dillon et al., 2015) and a smaller attentional window (Basso et al., 1996; de Fockert \& Cooper, 2014; Derryberry \& Tucker, 1994). If the attentional window is smaller, then these individuals might simply engage in searching for the target in a manner that is less sensitive to display factors (Müller et al., 2009). For instance, they might look for the target in a systematic fashion, moving the attentional window around the search array (e.g., starting at the top location in the search array and then moving clockwise). In this case, it would be expected that the level of interference by a foil presented at fixation would be more comparable across the two perceptual load conditions. If increasing depression symptoms diminishes the load effect when anxious arousal is low, why would the load effect increase when both depression and anxious arousal scores are elevated (see Fig. 4b)? As proposed by the attentional control theory, anxious arousal is associated with greater sensitivity to bottom-up information (Derakshan \& Eysenck, 2009; Eysenck et al., 2007). It follows that this tendency to overly process perceptual information would tend to counteract the impact of depression on perceptual processing, allowing for a more typical perceptual load effect to reemerge.

Note that this is not the first study to report a counterintuitive interaction between depression and anxiety on executive control. At the behavioral level, Snyder et al. (2014) demonstrated opposite effects of anxiety and depression on verbal selection tasks. The authors found that when controlling for anxiety, depression was associated with improved selection, and that when controlling for depression, anxiety was associated with impaired selection. At the neural level, depression and anxiety symptoms have been associated with divergent activation patterns in similar brain regions during tasks of attentional and inhibitory control (e.g., Engels et al., 2007; Engels et al., 2010; Warren et al., 2013).

For foils presented in the periphery, the most parsimonious model was the null model containing only the effect of load. Contrary to previous findings indicating that anxiety levels affected peripheral foil interference (Moriya \& Tanno, 2010), the maximum-likelihood model selection approach suggested that in our sample, levels of depression and anxiety levels did not contribute meaningfully to account for the observed data above and beyond the factor of load. It is possible that with peripheral foils, participants were faced with a somewhat easier selection task, as evidenced by faster response times and smaller interference effects (see Table 4). Indeed, interference effects were more than twice as large in magnitude for foils presented centrally than for those presented peripherally (replicating Beck \& Lavie, 2005). This result suggests that the differential effects of depression and anxiety dimensions were more readily observed when the foils were presented at fixation and were more difficult to ignore and suppress (Beck \& Lavie, 2005). Indeed, centrally presented foils caused an overall slowing of response times (see Table 4), which was even present on congruent trials, indicating that participants were actively suppressing central foil processing throughout the block of trials.

Finally, models containing anxious apprehension did not significantly predict interference for central nor peripheral 
foils. Anxious apprehension is characterized by thoughts about diffuse, future-oriented threats that are considered more verbal in nature when compared with somatic hyperarousal (i.e., anxious arousal; Engels et al., 2007; Hur et al., 2016; Nitschke et al., 2001; Nolen-Hoeksema, 2008). Anxious apprehension has been shown to be associated with increased activation in brain regions associated with verbal processing (e.g., left DLPFC, left IFG) in response to negatively valenced words (Engels et al., 2007; Warren et al., 2013). As the present study used nonemotional, nonword stimuli, it is possible that networks associated with verbal threat processing did not contribute to the active inhibition of foils. Overall, the results presented here highlight the importance of evaluating different types of anxiety, as only anxious arousal appeared to play a role along with depression on impacting attentional selection and response inhibition in the central foil interference task.

\section{Dimensional versus categorical effects}

The literatures discussed in the introduction regarding the effects of anxiety and depression symptoms on attentional performance used a categorical approach, such that participants were grouped as a function of whether they exhibited high or low scores on a scale. For instance, Dillon et al. (2015) compared clinically depressed individuals with nondepressed individuals. In the anxiety literature, a median split procedure was often used to separate low and high anxious individuals with respect to social anxiety (Moriya \& Tanno, 2010), trait anxiety (Sadeh \& Bredemeier, 2011), and generalized anxiety disorder (Najmi et al., 2015). As the present study evaluated anxious arousal and anxious apprehension, it seemed relevant to conduct similar analyses on the present data.

We conducted a post hoc analysis on the central foil data, with a categorical approach where participants were grouped into high and low groups, as a function of their $Z$ score (positive and negative values, respectively) on the corresponding scale, so that we could better compare our data to previously published categorical findings. We conducted the analyses on anxious arousal as well as on anxious apprehension (while ignoring depression scores). None of these analyses showed a significant group by load interaction, $F(1,151)=0.565, p=$ .454 , and $F(1,151)=0.351, p=.554$. Note that our dimensional analysis had also failed to observe a main effect of anxiety or an interaction between anxiety and load. Anxious arousal only modulated the interaction between depression and load.

Regarding depression, it is important to remember that our sample was not clinically diagnosed, like the one described in Dillon et al. (2015). For comparison, we created two groups at the two ends of the depression scale (disregarding anxiety scores). The high depression group included individuals who scored higher than the 80th percentile $(z$ score $>0.842, N=$ 31 ), and the low depression group included individuals scoring below the mean on the scale ( $z$ score $<0, N=83$, corresponding to the lower 54th percentile). The practice of selecting these asymmetric cutoffs is consistent with previous clinical research examining group differences in psychopathology dimensions (Bredemeier et al., 2010; Nitschke et al., 1999), with the high scoring group more clearly reflecting individuals with clinically significant depression symptoms and the low scoring group more clearly reflecting a not-atrisk for depression group. Note that the asymmetric selection has been shown to not significantly inflate effect sizes over a symmetrical cutoff approach (see Fisher, Guha, Heller, \& Miller, 2019). The Load $\times$ Group interaction was significant, $F(1,112)=4.33, p=.04$. Follow-up $t$ tests indicated that in the low perceptual load condition, flanker interference was smaller in the low depression group (1.09) than in the high depression group (1.13), $t(112)=-2.11, p=.037$. In the high perceptual load condition, similar flanker interference was observed in the two groups (1.081 vs. 1.078), $t(112)=0.190, p$ $=.85$. This finding indicates that the results reported in Dillon et al. (2015) replicate in a nonclinical population, when individuals scoring higher than the 80th percentile are considered. Interestingly, $60 \%$ of these individuals scored below the mean in the anxious arousal scale.

Using a median split procedure to analyze the effects of high or low depression or anxiety symptoms is common within the literature as reviewed above, although this methodological approach is not without limitations. For example, grouping participants based on whether a symptom score is above or below a cutoff value, or plus or minus one standard deviation from the mean does not account for the continuous nature of the scale and may result in overlooking an important subset of participants falling between the cutoff points. In addition, grouping participants based on diagnostic categories (e.g., participants with clinical depression vs. no depression) does not necessarily account for the level of symptom severity within groups or between participants (see Brown \& Barlow, 2005). Furthermore, studies that use a categorical approach to understand the effects of psychopathology symptoms on attention abilities may not systematically take into account the effects of co-occurring symptoms (e.g., depression and anxiety dimensions) on outcome variables. This is especially important given the high comorbidity across various psychological disorders, such as depression and anxiety (Kessler et al., 2011; Rusico \& Khazanov, 2017). In contrast, using several symptom scores as continuous, dimensional measures of psychopathology may better capture within-subject and betweensubjects variability and allow researchers to control for the effects of co-occurring symptoms. Of note, the issue regarding the use of categorical and dimensional approaches to understanding psychopathology symptoms and clinical disorders has been widely debated (see Brown \& Barlow, 2005; Widiger \& Samuel, 2005), and it has been suggested that the decision to use a dimensional or categorical approach to 
studying symptoms of psychopathology should be empirically informed and addressed at all levels of study design and analysis (Pickles \& Angold, 2003).

\section{Limitations}

Limitations to the present study should be noted. First, the sample included only college students. Further examination of attentional control with older as well as younger populations would add to the understanding of how response interference varies in the presence of depression and anxiety across the life span. Although the use of dimensional measures of depression and anxiety allowed for a sensitive assessment along a continuum of symptoms, it is possible that a sample with clinical diagnoses of depression or anxiety might have revealed stronger associations between the variables of interest. Future research that incorporates categorically defined groups and dimensional measures may help to clarify the results presented here. Finally, participants were not screened for medication use, which may also impact attentional abilities.

\section{Implications}

Little research to date has examined the effects of cooccurring symptoms of depression and anxiety on interference and prepotent response inhibition in the context of flanker task paradigms, and future studies should consider which specific attentional biases or control mechanisms contribute the most to interference in various flanker and real-world paradigms. As the lifetime prevalence of a mood disorder is $20.8 \%$ (Kessler et al., 2005), nonclinical researchers should be increasingly aware that variation in task performance within participant samples might be compromised by behavior patterns or symptoms that indicate psychological distress. These considerations are likely novel and unfamiliar to researchers from other fields within psychology. Without an adequate knowledge of symptom variation or dimensional models of depression and anxiety, the potential for researchers to misinterpret data and make incorrect assumptions about attentional processing within groups increases, particularly for studies using small samples (as is usually the case in attention studies, where sample size is often around 20). The use of brief selfreports of depression and anxiety dimensions, as well as other symptom measures, provides an ample starting point for these endeavors, provided researchers are willing to consider the importance of affect and mood symptoms within their own selective attention paradigms.

\section{Conclusion}

The present study used a dimensional assessment of psychopathology in a large sample of undergraduates who were not selected for particular symptom expressions. The use of the flanker paradigm reported here used nonemotional stimuli, thus removing potential confounds related to attentional biases for negatively valenced information that appear among individuals with higher levels of depression or anxiety. The findings suggested that individuals who scored low on both depression and anxious arousal scales exhibited a normal perceptual load effect on flanker interference (larger interference on low load compared with high load displays). Furthermore, a reduced perceptual load effect was observed in individuals who scored high on depression (and low in anxious arousal). Finally, the perceptual load effect reemerged in individuals who scored high on both depression and anxious arousal scales, but to a lesser extent than those scoring low in both. This is a significant finding given the high comorbidity of these two symptoms in the general population, and it highlights the importance of fully assessing the contributions and interactions of both symptoms on attentional performance. The results also suggest that samples similar to the one reported here are actually quite diverse regarding trait presentation, attentional processing, and inhibitory abilities.

Acknowledgments S.B., W.H., and A.W. designed the study. S.B. and A.W. wrote the paper, while W.H. gave feedback on the manuscript. A.W. ran the experiment and processed the data. Q.L. and S.B. did the analyses.

Author note This research received no specific grant from funding agencies in the public, commercial, or not-for-profit sectors. The authors report no conflicts of interest.

Open practices statement None of the data or materials for the experiment reported here is available, and the experiment was not preregistered.

\section{Compliance with ethical standards}

Ethical approval All procedures performed involving human participants were in accordance with the ethical standards of the institutional research committee and with the 1964 Helsinki declaration and its later amendments.

\section{References}

Ahmed, L., \& de Fockert, J. W. (2012). Focusing on attention: The effects of working memory capacity and load on selective attention. PLOS ONE, 7(8). https://doi.org/10.1371/journal.pone.0043101

Baert, S., De Raedt, R., \& Koster, E. (2010). Depression-related attentional bias: The influence of symptom severity and symptom specificity. Cognition \& Emotion, 24(6), 1044-1052. https://doi.org/10. 1080/02699930903043461

Bar-Haim, Y., Lamy, D., Pergamin, L., Bakermans-Kranenburg, M. J., \& van IJzendoorn, M. H. (2007). Threat-related attentional bias in anxious and nonanxious individuals: A meta-analytic study. Psychological Bulletin, 133(1), 1-24. https://doi.org/10.1037/ 0033-2909.133.1.1

Basso, M. R., Schefft, B. K., Ris, M. D., \& Dember, W. N. (1996). Mood and global-local visual processing. Journal of the International 
Neuropsychological Society : JINS, 2(3), 249-255. https://doi.org/ $10.1017 / \mathrm{S} 1355617700001193$

Beck, D. M., \& Lavie, N. (2005). Look here but ignore what you see: Effects of distractors at fixation. Journal of Experimental Psychology. Human Perception and Performance, 31(3), 592-607. https://doi.org/10.1037/0096-1523.31.3.592

Belopolsky, A. V, \& Theeuwes, J. (2010). No capture outside the attentional window. Vision Research, 50(23), 2543-2550. https://doi.org/ 10.1016/j.visres.2010.08.023

Belopolsky, A. V, Zwann, L., Theeuwes, J., \& Kramer, A. F. (2007). The size of an attentional window modulates attentional capture by color singletons. Psychonomic Bulletin \& Review, 14(5), 934-938.

Bolger, N., Zee, K. S., Rossignac-Milon, M., \& Hassin, R. R. (2019). Causal processes in psychology are heterogeneous. Journal of Experimental Psychology: General. https://doi.org/10.1037/ xge0000558

Bishop, S. J. (2009). Trait anxiety and impoverished prefrontal control of attention. Nature Neuroscience, 12(1), 92-98. https://doi.org/10. 1038/nn.2242

Bradley, B. P., Mogg, K., \& Lee, S. C. (1997). Attentional biases for negative information in induced and naturally occurring dysphoria. Behaviour Research and Therapy, 35(10), 911-927. https://doi.org/ 10.1016/S0005-7967(97)00053-3

Brainard, D. H. (1997). The Psychophysics Toolbox. Spatial Vision, 10, 433-436. https://doi.org/10.1163/156856897X00357

Bredemeier, K., Spielberg, J., Silton, R., Berenbaum, H., Heller, W., \& Miller. (2010). Screening for depressive disorders using the MASQ Anhedonic Depression scale: A receiver-operator characteristic analysis. Psychological Assessment, 22(3), 702-710. https://doi. org/10.1037/a0019915.Screening

Brown, T. A., Antony, M. M., \& Barlow, D. H. (1992). Psychometric properties of the Penn State Worry Questionnaire in a clinical anxiety disorders sample. Behaviour Research and Therapy, 30(1), 33 37. https://doi.org/10.1016/0005-7967(92)90093-V

Brown, T. A., \& Barlow, D. H. (2005). Dimensional versus categorical classification of mental disorders in the fifth edition of the Diagnostic and Statistical Manual of Mental Disorders and beyond: Comment on the special section. Journal of Abnormal Psychology, 114(4), 551-556. https://doi.org/10.1037/0021-843X.114.4.551

Buetti, S., Lleras, A., \& Moore, C. M. (2014). The flanker effect does not reflect the processing of "task-irrelevant" stimuli: Evidence from inattentional blindness. Psychonomic Bulletin \& Review, 12311237. https://doi.org/10.3758/s13423-014-0602-9

Chan, D., Rajsic, J., \& Pratt, J. (2017). Go-getters and procrastinators: Investigating individual differences in visual cognition across university semesters. Vision Research, 141, 317-324. https://doi.org/ 10.1016/j.visres.2016.09.007

Chiu, P. H., \& Deldin, P. (2007). Neural evidence for enhanced error detection in major depressive disorder. The American Journal of Psychiatry, 164(4), 608-616.

Clark, L., \& Watson, D. (1991). Tripartite model of anxiety and depression: Psychometric evidence and taxonomic implications. Journal of Abnormal Psychology, 100, 316-336. https://doi.org/10.1037/0021843X.100.3.316

Cohen, J. (1983). The cost of dichotomizaiton. Applied Psychological Measurement, 7, 247-253. https://doi.org/10.1177/ 014662168300700301

Crocker, L., Heller, W., Spielberg, J., Warren, S., Bredemeier, K., Sutton, B., ... Miller, G. (2012). Neural mechanisms of attentional control differentiate trait and state negative affect. Frontiers in Psychology, 3, 298. https://doi.org/10.3389/fpsyg.2012.00298

Dawson, J. F. (2014). Moderation in management research: What, why, when, and how. Journal of Business and Psychology, 29(1), 1-19. https://doi.org/10.1007/s10869-013-9308-7

Dawson, J. F., \& Richter, A. W. (2006). Probing three-way interactions in moderated multiple regression: Development and application of a slope difference test. The Journal of Applied Psychology, 91(4), 917-926. https://doi.org/10.1037/0021-9010.91.4.917

de Fockert, J. W., \& Cooper, A. (2014). Higher levels of depression are associated with reduced global bias in visual processing. Cognition and Emotion. https://doi.org/10.1080/02699931.2013.839939

Derakshan, N., \& Eysenck, M. (2009). Anxiety, processing efficiency, and cognitive performance: New developments from attentional control theory. European Psychologist, 14, 168-176.

Derryberry, D., \& Reed, M. A. (2002). Anxiety-related attentional biases and their regulation by attentional control. Journal of Abnormal Psychology, 111(2), 225-236. https://doi.org/10.1037/0021-843X. 111.2.225

Derryberry, D., \& Tucker, D. M. (1994). Motivating the focus of attention. In P. M. Niedenthal \& S. Kitayama (Eds.), In the heart's eye: Emotional influences in perception and attention (pp. 167-196). San Diego, CA: Academic Press.

Desimone, R., \& Duncan, J. (1995). Neural mechanisms of selective visual attention. Annual Review of Neuroscience, 18, 193-222.

Dillon, D. G., Wiecki, T., Pechtel, P., Webb, C., Goer, F., Murray, L., ... Pizzagalli, D. A. (2015). A computational analysis of flanker interference in depression. Psychological Medicine, 45(11), 2333-2344. https://doi.org/10.1017/S0033291715000276

Dubal, S., \& Jouvent, R. (2004). Time-on-task effect in trait anhedonia. European Psychiatry, 19(5), 285-291.

Dubal, S., Pierson, A., \& Jouvent, R. (2000). Focused attention in anhedonia: A P3 study. Psychophysiology. https://doi.org/10.1017/ S0048577200001943

Ebersole, C. R., Atherton, O. E., Belanger, A. L., Skulborstad, H. M., Allen, J. M., Banks, J. B., ... Nosek, B. A. (2016). Many Labs 3: Evaluating participant pool quality across the academic semester via replication. Journal of Experimental Social Psychology, 67, 68-82. https://doi.org/10.1016/j.jesp.2015.10.012

Engels, A. S., Heller, W., Mohanty, A., Herrington, J. D., Banich, M. T., Webb, A. G., \& Miller, G. A. (2007). Specificity of regional brain activity in anxiety types during emotion processing. Psychophysiology, 44(3), 352-363. https://doi.org/10.1111/j.14698986.2007.00518.x

Engels, A. S., Heller, W., Spielberg, J. M., Warren, S. L., Sutton, B. P., Banich, M. T., \& Miller, G. A. (2010). Co-occuring anxiety influences patterns of brain activity in depression. Cognitive, Affective, \& Behavioral Neuroscience, 10(1), 141-156. https://doi.org/10.1002/ cncr.29075.Familial

Eriksen, B. A., \& Eriksen, C. W. (1974). Effects of noise letters upon the identification of a target letter in a nonsearch task. Perception \& Psychophysics, 16(1), 143-149. https://doi.org/10.3758/ BF03203267

Eriksen, C. W., \& St James, J. D. (1986). Visual attention within and around the field of focal attention: A zoom lens model. Perception \& Psychophysics, 40(4), 225-240. https://doi.org/10.3758/ $\mathrm{BF} 03211502$

Eysenck, M., Derakshan, N., Santos, R., \& Calvo, M. (2007). Anxiety and cognitive performance: Attentional control theory. Emotion, 7(2), 336-353. https://doi.org/10.1037/1528-3542.7.2.336

Fisher, J. E., Guha, A., Heller, W., \& Miller, G. A. (2019). Extremegroups designs in studies of dimensional phenomena: Advantages, caveats, and recommendations. Journal of Abnormal Psychology. Advance online publication. https://doi.org/10.1037/abn0000480

Forster, S., \& Lavie, N. (2008). Attentional capture by entirely irrelevant distractors. Visual Cognition, 16(2/3), 200-214. https://doi.org/10. 1080/13506280701465049

Forster, S., \&Lavie, N. (2007). High Perceptual Load Makes Everybody Equal. Psychological Science, 18(5), 377-381

Forster, S., Robertson, D. J., Jennings, A., Asherson, P., \& Lavie, N. (2014). Plugging the attention deficit: Perceptual load counters increased distraction in ADHD. Neuropsychology, 28(1), 91-97. https://doi.org/10.1037/neu0000020 
Fredrickson, B. L., \& Branigan, C. (2005). Positive emotions broaden the scope of attention and thought-action repertoires. Cognition \& Emotion, 19(3), 313-332. https://doi.org/10.1080/ 02699930441000238

Fresco, D. M., Frankel, A. N., Mennin, D. S., Turk, C. L., \& Heimberg, R. G. (2002). Distinct and overlapping features of rumination and worry: The relationship of cognitive production to negative affective states. Cognitive Therapy and Research, 26(2), 179-188. https:// doi.org/10.1023/A:1014517718949

Fresco, D. M., Mennin, D. S., Heimberg, R. G., \& Turk, C. L. (2003). Using the Penn State Worry Questionnaire to identify individuals with generalized anxiety disorder: A receiver operating characteristic analysis. Journal of Behavior Therapy and Experimental Psychiatry, 34(3/4), 283-291. https://doi.org/10.1016/j.jbtep.2003. 09.001

Gasper, K. (2004). Do you see what I see? Affect and visual information processing. Cognition \& Emotion, 18(3), 405-421. https://doi.org/ 10.1080/02699930341000068

Goldberg, L. R., Johnson, J. A., Eber, H. W., Hogan, R., Ashton, M. C., Cloninger, C. R., \& Gough, H. G. (2006). The international personality item pool and the future of public-domain personality measures. Journal of Research in Personality, 40, 84-96. https://doi. org/10.1016/j.jrp.2005.08.007

Gotlib, I. H., \& Joormann, J. (2010). Cognition and depression: Current status and future directions. Annual Review of Clinical Psychology, 6, 285-312. https://doi.org/10.1146/annurev.clinpsy.121208. 131305

Gotlib, I. H., Krasnoperova, E., Neubauer Yue, D., \& Joormann, J. (2004). Attentional biases for negative interpersonal stimuli in clinical depression. Journal of Abnormal Psychology, 113(1), 127-135. https://doi.org/10.1037/0021-843X.113.1.127

Grimm, L. R., Markman, A. B., \& Maddox, W. T. (2012). End-ofsemester syndrome: How situational regulatory fit affects test performance over an academic semester. Basic and Applied Social Psychology, 34(4), 376-385. https://doi.org/10.1080/01973533. 2012.693427

Heller, W., Nitschke, J. B., Etienne, M. A., \& Miller, G. A. (1997). Patterns of regional brain activity differentiate types of anxiety. Journal of Abnormal Psychology, 106(3), 376-385. https://doi.org/ 10.1037/0021-843X.106.3.376

Holmes, A. J., \& Pizzagalli, D. A. (2010). Effects of task-relevant incentives on the electrophysiological correlates of error processing in major depressive disorder. Cognitive, Affective, \& Behavioral Neuroscience, 10(1), 119-128.

Humphreys, L. G. (1978). Doing research the hard way: Substituting analysis of variance for a problem in correlational analysis. Journal of Educational Psychology, 70(6), 873-876.

Humphreys, L. G., \& Fleishman, A. (1974). Pseudo-orthogonal and other analysis of variance designs involving individual-differences variables. Journal of Educational Psychology. https://doi.org/10.1037/ h0036539

Hur, J., Heller, W., Kern, J. L., \& Berenbaum, H. (2016). A Bi-Factor Approach to Modeling the Structure of Worry and Rumination. Journal of Experimental Psychology, 8(3), 252-264.

Iordan, A., Dolcos, S., \& Dolcos, F. (2013). Neural signatures of the response to emotional distraction: A review of evidence from brain imaging investigations. Frontiers in Human Neuroscience, 7(June), 200. https://doi.org/10.3389/fnhum.2013.00200

Joormann, J., \& Quinn, M. E. (2014). Cognitive processes and emotion regulation in depression. Depression and Anxiety, 31(4), 308-315. https://doi.org/10.1002/da.22264

Joormann, J., \& Vanderlind, W. M. (2014). Emotion regulation in depression: The role of biased cognition and reduced cognitive control. Clinical Psychological Science, 2(4), 402-421. https://doi.org/10. 1177/2167702614536163
Kessler, R. C., Berglund, P., Demler, O., Jin, R., Merikangas, K. R., \& Walters, E. E. (2005). Lifetime prevalence and age-of-onset distributions of DSM-IV disorders in the national comorbidity survey replication. Archives of General Psychiatry, 62(6), 593-602. https://doi.org/10.1001/archpsyc.62.6.593

Kessler, R. C., Ormel, J., Petukhova, M., McLaughlin, K. A, Green, J. G., Russo, L. J., ... Ustün, T. B. (2011). Development of lifetime comorbidity in the World Health Organization world mental health surveys. Archives of General Psychiatry, 68(1), 90-100. https:// doi.org/10.1001/archgenpsychiatry.2010.180

Lachter, J., Forster, K. I., \& Ruthruff, E. (2004). Forty-five years after Broadbent (1958): Still no identification without attention. Psychological Review, 111(4), 880-913. https://doi.org/10.1037/ 0033-295X.111.4.880

Lavie, N. (1995). Perceptual load as a necessary condition for selective attention. Journal of Experimental Psychology: Human Perception and Performance, 21(3), 451-468. https://doi.org/10.1037/00961523.21.3.451

Lavie, N. (2005). Distracted and confused?: Selective attention under load. Trends in Cognitive Sciences, 9(2), 75-82. https://doi.org/10. 1016/j.tics. 12.004

Lavie, N. (2010). Attention, Distraction, and Cognitive Control Under Load. Current Directions in Psychological Science, 19(3), 143148. https://doi.org/10.1177/0963721410370295

Lavie, N., \& Tsal, Y. (1994). Perceptual load as a major determinant of the locus of selection in visual attention. Perception \& Psychophysics, 56(2), 183-197. https://doi.org/10.3758/BF03213897

Lavie, N., Hirst, A., de Fockert, J. W., \& Viding, E. (2004). Load theory of selective attention and cognitive control. Journal of Experimental Psychology. General, 133(3), 339-354. https://doi.org/10.1037/ 0096-3445.133.3.339

Levin, R. L., Heller, W., Mohanty, A., Herrington, J. D., \& Miller, G. A. (2007). Cognitive deficits in depression and functional specificity of regional brain activity. Cognitive Therapy and Research, 31(2), 211-233. https://doi.org/10.1007/s10608-007-9128-z

Lleras, A., Buetti, S., \& Mordkoff, J. T. (2014). When do the effects of distractors provide a measure of distractibility? Psychology of Learning and Motivation-Advances in Research and Theory (Vol. 59). https://doi.org/10.1016/B978-0-12-407187-2.00007-1

MacCallum, R. C., Zhang, S., Preacher, K. J., \& Rucker, D. D. (2002). On the practice of dichotomization of quantitative variables. Psychological Methods. https://doi.org/10.1037/1082-989X.7.1.19

Max, R., \& Tsal, Y. (2015). The mutations paradigm: Assessing the time course of distractor processing. Attention, Perception, \& Psychophysics, 77(7), 2344-2355. https://doi.org/10.3758/s13414015-0928-Z

Maxwell, S. E., \& Delaney, H. D. (1993). Bivariate median splits and spurious statistical significance. Psychological Bulletin. https://doi. org/10.1037/0033-2909.113.1.181

Meyer, T. J., Miller, M. L., Metzger, R. L., \& Borkovec, T. D. (1990). Development and validation of the Penn State Worry Questionnaire. Behaviour Research and Therapy, 28, 487-495. https://doi.org/10. 1016/0005-7967(90)90135-6

Mogg, K., \& Bradley, B. P. (2005). Attentional bias in generalized anxiety disorder versus depressive disorder. Cognitive Therapy and Research, 29(1), 29-45. https://doi.org/10.1007/s10608-0051646-y

Moran, T. P. (2016). Anxiety and working memory capacity: A metaanalysis and narrative review. Psychological Bulletin, 142(8), 831864. https://doi.org/10.1037/bul0000051

Moriya, J., \& Tanno, Y. (2010). Attentional resources in social anxiety and the effects of perceptual load. Cognition and Emotion, 24(8), 1329-1348. https://doi.org/10.1080/02699930903378503

Müller, J., Wilmsmann, D., Exeler, J., Buzeck, M., Schmidt, A., Jay, T., \& Krüger, A. (2009). Display blindness: The effect of expectations on attention towards digital signage. In H. Tokuda, M. Beigl, A. Friday, 
A. J. B. Brush, \& Y. Tobe (Eds.), International conference on pervasive computing (pp. 1-8). Berlin, Germany: Springer. https://doi. org/10.1007/978-3-642-01516-8 1

Najmi, S., Amir, N., Frosio, K. E., \& Ayers, C. (2015). The effects of cognitive load on attention control in subclinical anxiety and generalised anxiety disorder. Cognition and Emotion, 29(7), 1210-1223. https://doi.org/10.1080/02699931.2014.975188

Nicholls, M. E. R., Loveless, K. M., Thomas, N. A., Loetscher, T., \& Churches, O. (2015). Some participants may be better than others: Sustained attention and motivation are higher early in semester. Quarterly Journal of Experimental Psychology, 68(1), 10-18. https://doi.org/10.1080/17470218.2014.925481

Nitschke, J., Heller, W., Imig, J., McDonald, R., \& Miller, G. (2001). Distinguishing dimensions of anxiety and depression. Cognitive Therapy and Research, 25(1), 1-22. https://doi.org/10.1023/A: 1026485530405

Nitschke, J., Heller, W., Palmieri, P., \& Miller, G. (1999). Contrasting patterns of brain activity in anxious apprehension and anxious arousal. Psychophysiology, 36(5), 628-637. https://doi.org/10.1017/ S0048577299972013

Nolen-Hoeksema, S. (2008). Rethinking rumination. Perspectives on Psychological Science, 3(5). 400-424. https://doi.org/10.1111/j. 1745-6924.2008.00088.x

Pelli, D. G. (1997). The VideoToolbox software for visual psychophysics: Transforming numbers into movies. Spatial Vision, 10(4), 437-442. https://doi.org/10.1163/156856897X00366

Peters, C. C., \& Van Voorhis, W. R. (1940). Statistical procedures and their mathematical bases. New York, NY: McGraw Hill.

Pickles, A., \& Angold, A. (2003). Natural categories or fundamental dimensions: On carving nature at the joints and the rearticulation of psychopathology. Development and Psychopathology, 15(3), 529-551.

Pizzagalli, D., \& Holmes, A. (2009). Reduced caudate and nucleus accumbens response to rewards in unmedicated subjects with major depressive disorder. The American Journal of Psychiatry, 66(6), 702-710. https://doi.org/10.1176/appi.ajp.2008.08081201

Rowe, G., Hirsh, J., \& Anderson, A. (2007). Positive affect increases the breadth of attentional selection. Proceedings of the National Academy of Sciences of the United States of America, 104(1), 383-388. https://doi.org/10.1073/pnas.0605198104

Rusico, A. M., \& Khazanov, G. K. (2017). Anxiety and depression. In R. J. DeRubeis \& D. R. Strunk (Eds.), The Oxford handbook of mood disorders (pp. 313-324). Oxford, England: Oxford University Press.

Sadeh, N., \& Bredemeier, K. (2011). Individual differences at high perceptual load: The relation between trait anxiety and selective attention. Cognition \& Emotion, 25(4), 747-755. https://doi.org/10.1080/ 02699931.2010 .500566

Sharp, P. B., Miller, G. A., \& Heller, W. (2015). Transdiagnostic dimensions of anxiety: Neural mechanisms, executive functions, and new directions. International Journal of Psychophysiology, 98(2), 365377. https://doi.org/10.1016/j.ijpsycho.2015.07.001
Siegle, G. J., Steinhauser, S. R., \& Thase, M. E. (2004). Pupillary assessment and computational modeling of the Stroop task in depression. International Journal of Psychophysiology, 52(1), 63-76.

Snyder, H. R. (2013). Major depressive disorder is associated with broad impairments on neuropsychological measures of executive function: A meta-analysis and review. Psychological Bulletin, 139(1), 81132. https://doi.org/10.1037/a0028727

Snyder, H. R., Kaiser, R. H., Whisman, M. A., Turner, A. E. J., Guild, R. M., \& Munakata, Y. (2014). Opposite effects of anxiety and depressive symptoms on executive function: The case of selecting among competing options. Cognition \& Emotion, 28(5), 893-902. https:// doi.org/10.1080/02699931.2013.859568

Spielberg, J. M., Miller, G. A., Warren, S. L., Engels, A. S., Crocker, L. D., Banich, M. T., ... Heller, W. (2012). A brain network instantiating approach and avoidance motivation. Psychophysiology, 49(9), 1200-1214. https://doi.org/10.1111/j.1469-8986.2012.01443.x

Tsal, Y., \& Benoni, H. (2010). Diluting the burden of load: Perceptual load effects are simply dilution effects. Journal of Experimental Psychology: Human Perception and Performance, 36(6), 16451656. https://doi.org/10.1037/a0018172

Vargha, A., Rudas, T., Delaney, H. D., \& Maxwell, S. E. (1996). Dichotomization, partial correlation, and conditional independence. Journal of Educational and Behavioral Statistics, 21(3), 264-282.

Wagenmakers, E.-J., \& Farrell, S. (2004). AIC model selection using Akaike weights. Psychonomic Bulletin \& Review, 11(1), 192-196. https://doi.org/10.3758/BF03206482

Warren, S. L., Crocker, L. D., Spielberg, J. M., Engels, A. S., Banich, M. T., Sutton, B. P., ... Heller, W. (2013). Cortical organization of inhibition-related functions and modulation by psychopathology. Frontiers in Human Neuroscience, 7(6), 271. https://doi.org/10. 3389/fnhum.2013.00271

Watson, D., Clark, L. A., Weber, K., Assenheimer, J. S., Strauss, M. E., \& McCormick, R. A. (1995). Testing a tripartite model: II. Exploring the symptom structure of anxiety and depression in student, adult, and patient samples. Journal of Abnormal Psychology, 104, 15-25. https://doi.org/10.1037/0021-843X.104.1.15

Watson, D., Weber, K., Assenheimer, J. S., Clark, L. A., Strauss, M. E., \& McCormick, R. A. (1995). Testing a tripartite model: I. Evaluating the convergent and discriminant validity of anxiety and depression symptom scales. Journal of Abnormal Psychology, 104, 3-14. https://doi.org/10.1037/0021-843X.104.1.3

Widiger, T. A., \& Samuel, D. B. (2005). Diagnostic categories or dimensions? A question for the Diagnostic and Statistical Manual of Mental Disorders-Fifth Edition. Journal of Abnormal Psychology, 114(4), 494-504. https://doi.org/10.1037/0021-843X.114.4.494

Publisher's note Springer Nature remains neutral with regard to jurisdictional claims in published maps and institutional affiliations. 